\title{
OPERADOR DE DUALIDAD Y ECUACIÓN NO-LINEAL ABSTRACTA DE KIRCHHOFF-CARRIER
}

\section{Raúl Izaguirre Maguiña ${ }^{1}$}

RESUMEN.- Sea $T>0$ y $\Omega \subset \mathbb{R}^{n}$ un subconjunto acotado con frontera regular $\Gamma=\partial \Omega$. En el presente trabajo demostramos que el problema de valor inicial

$$
\left(^{*}\right) \begin{cases}\rho(x) u_{t t}-M\left(|u(t)|_{p}\right) \Delta u=f & \text { en } \Omega \times(0, T) \\ u(x, t)=0 & \text { en } \Gamma \times\left(0, T^{\prime}\right) \\ u(x, 0)=u_{0} \quad ; \quad u^{\prime}(x, 0)=u_{1} & \text { en } \Omega\end{cases}
$$

donde $|\cdot|_{p}$ denota la norma en el espacio $L^{p}(\Omega)$ y M es una función nonegativa de clase $C^{1}$, tiene una única solución local, bajo adecuadas condiciones sobre $\rho, M, f, u_{0}, u_{1}$.

PALABRAS CLAVE:- Ecuaciones Diferenciales parciales no lineales. Ecuación abstracta de Kirchhoff-Carrier.

\section{DUALITY OPERATOR AND ABSTRACT NONLINEAR EQUATION OF KIRCHHOFF-CARRIER}

ABSTRACT.- Let $T>0$ and $\Omega \subset \mathbb{R}^{n}$ be a bounded subset with regular boundary $\Gamma=\partial \Omega$. We prove that the initial value problem:

$$
\left(^{*}\right) \begin{cases}\rho(x) u_{t t}-M\left(|u(t)|_{p}\right) \Delta u=f & \text { on } \Omega \times(0, T) \\ u(x, t)=0 & \text { on } \Gamma \times(0, T) \\ u(x, 0)=u_{0} ; u^{\prime}(x, 0)=u_{1} & \text { on } \Omega\end{cases}
$$

where ||$_{p}$ denotes the $L^{p}(\Omega)$ norm and $M$ is a non-negative function under adequate conditions on $\rho, M, f, u_{0}, u_{1}$.

KEYWORDS.- Nonlinear partial differential equations. Abstract equation of Kirchhoff - Carrier.

\footnotetext{
'Universidad Nacional Mayor de San Marcos. Facultad de Ciencias Matemáticas. e-mail: rizaguirrem@umnsm.edu.pe
} 


\section{Introducción}

Sean $(V, a(u, v)),(H, b(u, v))$, espacios de Hilbert, $V \subset H$ la inmersión de $V$ en $H$ es densa y compacta. Sea también $A$ el operador definido por la terna $\{V, H, a(u, v)\}$. Entonces $A$ es un operador no-acotado, auto-adjunto y positivo de $H$, con espectro discreto. Asimismo para todo $\alpha \in \mathbb{R}$, el operador $A^{\alpha}$ está bien definido. En este contexto $V=D\left(A^{1 / 2}\right)$.

El siguiente modelo abstracto

$$
\left\{\begin{array}{l}
u^{\prime \prime}+M\left(\left|A^{\alpha} u\right|^{2}\right) A^{\beta} u=f \\
u(0)=u_{0} ; u^{\prime}(0)=u_{1}
\end{array}\right.
$$

considera como casos particulares los siguientes problemas

Sea $\Omega$ un abierto $\mathbb{R}^{n}$ con frontera regular $\Gamma ; Q$ el cilindro $\left.\Omega \times\right] 0, T[, 0<T<\infty$ con frontera lateral $\Sigma=\Gamma \times] 0, T]$. La ecuación diferencial parcial no-lineal

$$
\begin{cases}u_{t t}+\left(1+\int_{\Omega}|\nabla u|^{2} d x\right)(-\Delta u)=f(t) & \text { en } Q \\ u=0 & \text { en } \Sigma \\ u(0)=u_{0} ; u_{t}(0)=u_{1} & \text { en } \Omega\end{cases}
$$

es un modelo generalizado de la ecuación de Kirchoff [7], planteada para estudiar las vibraciones de pequeña amplitud, de una cuerda fija en sus extremos y cuando la dependencia de la tensión no puede dejarse de lado en el modelo.

Asimismo la ecuación

$$
\begin{cases}u_{t t}+\left(1+\int_{\Omega}|u|^{2} d x\right)(-\Delta u)=f(t) & \text { en } Q \\ u=0 & \text { en } \Sigma \\ u(0)=u_{0} ; u_{t}(0)=u_{1} & \text { en } \Omega\end{cases}
$$

Es una generalización de un problema estudiado por Carrier en [2].

En [14] Pohozaev trata el sistema

$$
\begin{cases}u^{\prime \prime}+(-1)^{m}\left(1+\int_{\Omega}\left|\nabla^{m} u\right|^{2} d x\right) \Delta^{m} u=f & \text { en } Q \\ \gamma_{0} u=\gamma_{1} u=\ldots \ldots \ldots . .=\gamma_{m-1} u=0 & \text { en } \Sigma \\ u(0)=u_{0} ; u^{\prime}(0)=u_{1} & \text { en } \Omega\end{cases}
$$


Obsérvese que si $V=H_{0}^{1}(\Omega), H=L^{2}(\Omega), A=-\Delta, \alpha=1 / 2, \beta=1$, estamos en la ecuación de Kirchoff (1.2); si $\alpha=0, \beta=1$, es el modelo de Carrier (1.3); si $V=H_{0}^{m}(\Omega), H=L^{2}(\Omega)$, $A=-\Delta ; \alpha=m / 2, \beta=m$, es el modelo de Pohozaev. En los casos señalados $M(s)=1+s^{2}$.

Con relación a esta formulación se plantea entre otros problemas el estudio de la ecuación (1.3) en el caso que la función no-lineal $M$ sea no-negativa (caso degenerado). La existencia y unicidad de soluciones locales para el caso degenerado de la ecuación de Kirchhoff $\alpha=1 / 2$ y $\beta=1$, son obtenidas por ejemplo en Ebihara - Medeiros - Milla [5], asumiendo que $M \in C^{l}$ y que $\left|M^{\prime}(s) s\right| \leq a M(s)$, donde la constante $a$ es positiva. En [4] Crippa trata un caso bastante general suponiendo que la función $M \in C^{1}([0, \infty)), M(0)=0 \mathrm{y} \forall \delta>0, \frac{\mathrm{inf}}{s \geq \delta} M(s)>0$ demostrando que existe una única solución local débil al problema de Cauchy asociado a (1.3).

En relación al modelo de Kirchoff - Carrier (1.3), se tiene las referencias [3] y [6], y donde la función no-lineal M es de clase $C^{1}$ y estrictamente positiva. En [3], se obtiene soluciones globales con datos analíticos "suficientemente pequeños". En [6] se obtiene solución local, pero en una clase mayor de datos iniciales. Para el caso degenerado se tiene la referencia [5] donde $\mathrm{M}$ es de clase $C^{\mathrm{l}}$ y verifica cierta condición de crecimiento polimonial.

Una generalización del modelo (1.4) consiste en el sistema

$$
K u^{\prime \prime}(t)+M\left(|B u(t)|^{2}\right) A u(t)=f(t)
$$

donde $K, B$ son operadores que conmutan con el operador $A$ y cumplen ciertos requisitos técnicos, referencia [7].

Otra manera de tratar unificadamente los sistemas (1.2), (1.3) y (1.4), es considerar el modelo

$$
u^{\prime \prime}(t)+M\left(\left|A^{\alpha} u(t)\right|^{2},\left|A^{\beta} u(t)\right|^{2}\right) A^{\gamma} u(t)=f(t)
$$

donde la función no-lineal $M(s, r)$ es no - negativa y de clase $C^{1}$ en las dos variables. Se demuestra la existencia de solución para el caso $\alpha=1 / 2, \beta=0 ; \gamma=1$ y en Izaguirre [6], se demuestra la existencia de solución para un caso más general.

El modelo abstracto (1), sin embargo, no incluye el tratamiento de la ecuación

$$
\begin{cases}\rho(x) u_{t t}+\left(1+\int_{\Omega}|u|^{p} d x\right)(-\Delta u)=f(t) & \text { en } Q \\ u=0 & \text { en } \Sigma \\ u(0)=u_{0} ; u_{t}(0)=u_{1} & \text { en } \Omega\end{cases}
$$

$1<p<\infty ; p \neq 2$. En el presente trabajo tratamos el problema de determinar solución local y unicidad de (5), modelo abstracto que incluye el problema (1.7). 
2. Preliminares.- Sea $(V, a(u, v))$ un espacio de Hilbert real; la forma bilineal $b: V \times V \rightarrow \mathbb{R}$ es simétrica, compacta, continua, estrictamente monótona y sea $H_{0}=(V, b(u, v))$, entonces $H_{0}$ es un espacio pre-Hilbert y podemos considerar su completación $H$ que es un espacio de Hilbert, con producto interno denotado por $b(u, v)$. Luego $V \subset H \subset V^{*}$ con inmersiones densas y continuas. La inmersión de $V$ en $H$ es compacta.

Consideramos entonces el operador $S: D(S) \rightarrow H$, tal que

$$
\begin{gathered}
D(S)=\{u \in V ; S u \in H\} \\
b(S u, v)=a(u, v)
\end{gathered}
$$

Entonces $S$ es un operador lineal, simétrico, no-acotado y estrictamente monótono desde que

$$
a(u, S u)=b(S u, S u)
$$

El operador inverso $S^{-1}: H \rightarrow D(S)$, es lineal, continuo, simétrico y compacto.

Formalmente se tiene que $S^{-1}=A^{-1} B$.

Por la teoría de los operadores compactos simétricos, tenemos que existe una base ortogonal y numerable $\left(w_{k}\right)_{k \geq 1}$ de $V$ y una sucesión creciente de números positivos $\left(\lambda_{k}\right)_{k \geq 1}$ tales que:

$$
\begin{aligned}
& \lambda_{j} b\left(w_{j}, v\right)=a\left(w_{j}, v\right) \quad \forall v \in V ; j=1,2, \ldots \ldots \\
& b\left(w_{j}, w_{i}\right)=\delta_{i j} ; a\left(w_{j}, w_{j}\right)=\lambda_{j} ; j=1,2, \ldots \ldots \\
& S w_{j}=\lambda_{j} w_{j} ; j=1,2, \ldots \ldots \ldots \\
& u=\sum_{j=1}^{\infty} b\left(u, w_{j}\right) w_{j} ; \forall u \in V
\end{aligned}
$$

De la teoría espectral para $\alpha \in \mathbb{R}$, podemos definir la potencia $S^{\alpha}$, con dominio

$$
\begin{aligned}
& D\left(S^{\alpha}\right)=\left\{u ; \sum_{v=1}^{\infty} \lambda_{v}^{2 \alpha}\left|b\left(u, w_{v}\right)\right|^{2}<\infty\right\} \\
& S^{\alpha} u=\sum_{v=1}^{\infty} \lambda_{v}^{\alpha} b\left(u, w_{v}\right) w_{v} \quad \forall u D\left(s^{\alpha}\right)
\end{aligned}
$$

Definimos entonces:

$$
\begin{aligned}
& b_{\alpha}(u, v)=b\left(S^{\alpha} u, S^{\alpha} v\right) \quad u, v \in D\left(S^{\alpha}\right) \\
& |u|_{\alpha}^{2}=b_{\alpha}(u, u) \quad u \in D\left(S^{\alpha}\right)
\end{aligned}
$$


se tiene que $\left(D\left(S^{\alpha}\right), b_{\alpha}(u, v)\right)$ es un espacio de Hilbert y si $\alpha<\beta$, la inmersión de $D\left(S^{\beta}\right) \subset D\left(S^{\alpha}\right)$ es compacta.

3. Problema Lineal. (Solución global). Sea $T>0$ y

$$
\begin{aligned}
& \psi \in C^{1}([0, T]), \psi(t) \geq m_{0}>0 \quad \forall s \in[0, T] \\
& \psi \in L^{\infty}(0, T) ;|\psi|_{L^{\infty}(0, T)} \leq M_{1} .
\end{aligned}
$$

Nos planteamos resolver el problema siguiente:

$$
\begin{aligned}
& B u^{\prime \prime}+\psi(t) A u=f \\
& u(0)=u_{0} ; u^{\prime}(0)=u_{1}
\end{aligned}
$$

Formalmente aplicando $A^{-1}$ en la ecuación (3.3)

$$
S^{-1} u^{\prime \prime}(t)+\psi(t) u(t)=S^{-1} B^{-1} f(t)=S^{-1} h(t)
$$

ahora aplicando en (3.5) $S^{\alpha+1}$ y considerando el producto interno en $L^{2}\left(0, T ; D\left(S^{\alpha}\right)\right)$ obtenemos

$$
\int_{0}^{T}\left[b_{\alpha}\left(u^{\prime \prime}(t), v\right)+\psi(t) b_{\alpha}(S u(t), v)\right] d t=\int_{0}^{T} b_{\alpha}(h(t), v) d t v \in L^{2}\left(0, T ; D\left(S^{\alpha}\right)\right)
$$

es en este sentido que entenderemos nuestra solución al problema (3.3) y (3.4).

Teorema 1. Sea $\alpha \in \mathbb{R}$ y $\psi$ que verifica las condiciones (3.1) y (3.2).

$$
\begin{aligned}
& u_{0} \in D\left(S^{\alpha+1}\right) \\
& u_{1} \in D\left(S^{(2 \alpha+1) / 2}\right) \\
& B^{-1} f=h \in L^{2}\left(0, T ; D\left(S^{(2 \alpha+1) / 2}\right)\right)
\end{aligned}
$$

Entonces existe una única solución $u$ del problema (3.3) y (3.4) tal que:

$$
\begin{aligned}
& u \in L^{\infty}\left(0, T ; D\left(S^{\alpha+1}\right)\right) \\
& u^{\prime} \in L^{\infty}\left(0, T ; D\left(S^{(2 \alpha+1) / 2}\right)\right) \\
& u^{\prime \prime} \in L^{2}\left(0, T ; D\left(S^{\alpha}\right)\right) \\
& B u^{\prime \prime}+\psi(t) A u=h \text { en } L^{2}\left(0, T ; D\left(S^{\alpha}\right)\right)
\end{aligned}
$$

La solución satisface el siguiente estimado de energía:

$$
E(t)=\left|S^{\alpha} u^{\prime}(t)\right|^{2}+\left|S^{2 \alpha+1 / 2} u(t)\right|^{2} \leq D_{0} \exp \left(D_{1} t\right)
$$

donde 


$$
\begin{aligned}
& C_{0}=\left|S^{(2 \alpha+1) / 2} u_{1}\right|^{2}+\psi(0)\left|S^{\alpha+1} u_{0}\right|^{2}+|h|_{L^{2}\left(0, T ; D\left(S^{(2 \alpha+1) / 2}\right)\right)}^{2} \\
& C_{1}=\min \left\{1, m_{0}\right\} ; \quad C_{2}=\operatorname{Max}\left\{1, M_{0}\right\} \\
& D_{0}=\frac{C_{0}}{C_{1}} ; D_{1}=\frac{C_{2}}{C_{1}}
\end{aligned}
$$

Demostración. Sea $V_{m}=\left[w_{1}, w_{2}, \ldots \ldots . . ., w_{m}\right] \subset V$. Luego $V_{m}$ es un subespacio de $V$ de dimensión finita $m$, e invariante bajo la acción del operador $S^{\alpha}, \alpha \in \mathbb{R}$.

Sea entonces

$$
u_{m}(t)=\sum_{i=1}^{m} g_{j m}(t) w_{i} \in V_{m}
$$

donde las funciones $g_{i m}$, son determinadas por la solución del siguiente sistema de ecuaciones diferenciales ordinarias lineales:

$$
\left\{\begin{array}{l}
b\left(u_{m}^{\pi \prime}(t), w_{j}\right)+\psi(t) a\left(u_{m}(t), w_{j}\right)=b\left(h(t), w_{j}\right) \quad \forall j=1,2, \ldots, m \\
u_{m}(0)=u_{0 m} ; u_{m}^{\prime}(0)=u_{1 m}
\end{array}\right.
$$

Por la selección de la base especial, el sistema es equivalente con el sistema lineal de ecuaciones diferenciales ordinarias:

$$
\left\{\begin{array}{l}
g_{j m}^{\prime \prime}(t)+\psi(t) \sum_{k=1}^{m} \lambda_{k} g_{k m}(t)=b\left(h(t), w_{j}\right)=h_{j}(t) \\
g_{j m}(0)=b\left(u_{0}, w_{j}\right)=a_{j} \\
g_{j m}^{\prime}(0)=b\left(u_{1}, w_{j}\right)=b_{j} \\
j=1,2, \ldots \ldots . ., m
\end{array}\right.
$$

El sistema de ecuaciones diferenciales lineales (3.17), admite una única solución en un intervalo $\left[0, t_{m}\right)$, de donde obtenemos la existencia de las soluciones aproximadas $u_{m}$ para $m \geq 1$. Seguidamente debemos obtener estimados a priori para la sucesión $\left\{u_{m}\right\}$ de modo que podamos prolongarlas a un intervalo uniforme de existencia.

Estimado a Priori 1. Por linealidad la ecuación en (3.16) se verifica para todo $v \in V_{m}$. Entonces haciendo $v=2 S^{2 \alpha+2} u_{m}^{\prime}(t)$, obtenemos:

$$
2 b\left(u_{m}^{\prime \prime}(t), S^{2 \alpha+2} u_{m}^{\prime}(t)\right)+2 \psi(t) a\left(u_{m}(t), S^{2 \alpha+2} u_{m}^{\prime}(t)\right)=2 b\left(h(t), S^{2 \alpha+2} u_{m}^{\prime}(t)\right)
$$

desde que

$$
\begin{aligned}
2 b\left(u_{m}^{\prime \prime}(t), S^{2 \alpha+2} u_{m}^{\prime}(t)\right)=2 a & \left(S^{-1} u_{m}^{\prime \prime}(t), S^{2 \alpha+2} u_{m}^{\prime}(t)\right)=\frac{d}{d t}\left|S^{(2 \alpha+1) / 2} u_{m}^{\prime}(t)\right|^{2} \\
2 \psi(t) a\left(u_{m}(t), S^{2 \alpha+2} u_{m}^{\prime}(t)\right) & =2 \psi(t) a\left(S^{\alpha+1} u_{m}(t), S^{\alpha+1} u_{m}^{\prime}(t)\right)= \\
& =\frac{d}{d t} \psi(s)\left|S^{\alpha+1} u_{m}(t)\right|^{2}-\psi^{\prime}(t)\left|S^{\alpha+1} u_{m}(t)\right|^{2}
\end{aligned}
$$


$2 b\left(h(t), S^{2 \alpha+2} u_{m}^{\prime}(t)\right)=2 a\left(S^{(2 \alpha+1) / 2} h(t), S^{(2 \alpha+1) / 2} u_{m}^{\prime}(t)\right) \leq\left|S^{(2 \alpha+1) / 2}(t)\right|^{2}\left|S^{(2 \alpha+1) / 2} u_{m}^{\prime}(t)\right|^{2}$

reemplazando en (3.19)

$\frac{d}{d t}\left\{\left|S^{(2 \alpha+1) / 2} u_{m}^{\prime}(t)\right|^{2}+\psi(t)\left|S^{\alpha+1} u_{m}(t)\right|^{2}\right\} \leq\left|S^{(2 \alpha+1) / 2} h(t)\right|^{2}+\left|S^{(2 \alpha+1) / 2} u_{m}^{+}(t)\right|^{2}+M_{1}\left|S^{\alpha+1} u_{m}(t)\right|^{2}(3.22)$

Ahora integrando en esta desigualdad:

$$
\begin{aligned}
& \left|S^{(2 \alpha+1) / 2} u_{m}^{\prime}(t)\right|^{2}+m_{0}\left|S^{\alpha+1} u_{m}(t)\right|^{2} \leq\left|S^{(2 \alpha+1) / 2} u_{m}^{\prime}(t)\right|^{2}+\psi(t)\left|S^{\alpha+1} u_{m}(t)\right|^{2} \leq \\
& \leq\left|S^{(2 \alpha+1) / 2} u_{m}^{\prime}(0)\right|^{2}+\psi(0)\left|S^{\alpha+1} u_{m}(0)\right|^{2}+\int_{0}^{t}\left|S^{(2 \alpha+1) / 2} h(s)\right|^{2} d s+\int_{0}^{t}\left|S^{(2 \alpha+1) / 2} u_{m}^{t}(s)\right|^{2} d s+ \\
& +M_{1} \int_{0}^{t}\left|S^{\alpha+1} u_{m}(s)\right|^{2} d s \leq C_{0}+C_{2} \int_{0}^{t} \tau(s) d s
\end{aligned}
$$

Aplicando el lema de Gronwall obtenemos:

$$
\left|S^{(2 \alpha+1) / 2} u_{m}^{\prime}(t)\right|^{2}+\left|S^{\alpha+1} u_{m}(t)\right|^{2} \leq \frac{C_{0}}{C_{1}} \exp \left\{C_{2} t / C_{1}\right\}=D_{0} \exp \left\{D_{1} t\right\}
$$

luego

$$
\begin{aligned}
& \left(u_{m}\right) \text { es acotada en } L^{\infty}\left(0, T ; D\left(S^{\alpha+1}\right)\right) \\
& \left(u_{m}^{\prime}\right) \text { es acotada en } L^{\infty}\left(0, T ; D\left(S^{(2 \alpha+1) / 2}\right)\right)
\end{aligned}
$$

Estimado a Prori 2. Reemplazando $w_{j}$ por $S^{\alpha} w_{j}$ en la ecuación aproximada y multiplicándola por $w_{j}$, obtenemos

$$
\sum_{j=1}^{m} b\left(u_{m}^{\prime \prime}(t), S^{\alpha} w_{j}\right) w_{j}+\psi(t) \sum_{j=1}^{m} a\left(u_{m}(t), S^{\alpha} w_{j}\right) w_{j}=\sum_{j=1}^{m} b\left(h(t), S^{\alpha} w_{j}\right) w_{j}
$$

Luego

$$
\begin{aligned}
& \sum_{j=1}^{m} b\left(u_{m}^{\prime \prime}(t), S^{\alpha} w_{j}\right)=\sum_{j=1}^{m} b\left(S^{\alpha} u_{m}^{\prime \prime}(t), w_{j}\right) w_{j}=S^{\alpha} u_{m}^{\prime \prime}(t)= \\
& =-\psi(t) \sum_{j=1}^{m} a\left(u_{m}(t), S^{\alpha} w_{j}\right) w_{j}+\sum_{j=1}^{m} b\left(h(t), S^{\alpha} w_{j}\right) w_{j}= \\
& =-\psi(t) \sum_{j=1}^{m} b\left(S u_{m}(t), S^{\alpha} w_{j}\right) w_{j}+\sum_{j=1}^{m} b\left(h(t), S^{\alpha} w_{j}\right) w_{j}= \\
& =-\psi(t) \sum_{j=1}^{m} b\left(S^{\alpha+1} u_{m}(t) w_{j}\right), w_{j}+\sum_{j=1}^{m} b\left(S^{\alpha} h(t), w_{j}\right) w_{j}= \\
& =-\psi(t) P_{m} S^{\alpha+1} u_{m}(t)+P_{m} h(t)=-\psi(t) S^{\alpha+1} u_{m}(t)+P_{m} h(t)
\end{aligned}
$$


Por lo tanto

$$
\left(u_{m}^{\prime \prime}\right) \text { es acotada en } L^{2}\left(0, T ; D\left(S^{\alpha}\right)\right)
$$

\section{Convergencia de las Soluciones Aproximadas}

Para tratar la convergencia de las soluciones aproximadas utilizamos el siguiente

Lema 1. Sean $X, Y, Z$ espacios de Banach tales que $X \subset Y \subseteq Z$, con inmersiones continuas y la inmersión $X \subset Y$ es compacta. Sea

$$
W=\left\{u \in L^{p}(0, T ; X) ; u^{\prime} \in L^{q}(0, T ; Z)\right\}
$$

donde $u^{\prime}$ denota la derivada generalizada de $u:[0, T] \rightarrow X$ sobre $(0, T)$.

$$
\begin{aligned}
& \text { Si } p=\infty, q>1 \text { entonces } W \subseteq C([0, T] ; Z) \text { es compacta. } \\
& \text { Si } 1 \leq p<\infty, q=1 \text { entonces } W \subseteq L^{p}(0, T ; Y) \text { es compacta. }
\end{aligned}
$$

Demostración. (Ver J. Simon [18]).

Por los estimados 1 y 2 , tenemos que:

$$
\begin{aligned}
& \left(u_{m}\right) \text { es acotada en } L^{\infty}\left(0, T ; D\left(S^{\alpha+1}\right)\right) \\
& \left(u_{m}^{\prime}\right) \text { es acotada en } L^{\infty}\left(0, T ; D\left(S^{(2 \alpha+1) / 2}\right)\right) \\
& \left(u_{m}^{\prime \prime}\right) \text { es acotada en } L^{2}\left(0, T ; D\left(S^{\alpha}\right)\right)
\end{aligned}
$$

Entonces, existe una subsucesión de $\left(u_{m}\right)$ que continuamos denotando de la misma forma tal que:

$$
\begin{aligned}
& u_{m} \rightarrow u \text { débil - * en } L^{\infty}\left(0, T ; D\left(S^{\alpha+1}\right)\right) \\
& u_{m}^{\prime \prime} \rightarrow u^{\prime \prime} \text { débil en } L^{2}\left(0, T ; D\left(S^{\alpha}\right)\right)
\end{aligned}
$$

Entonces:

$$
\begin{aligned}
& \int_{0}^{T}\left[b_{\alpha}\left(u^{\prime \prime}(t), v\right)+\psi(t) b_{\alpha}(S u(t), v)\right] \theta(t) d t=\int_{0}^{T} b_{\alpha}(h(t), v) \theta(t) d t ; \\
& \forall \theta \in D(0, T), \forall v \in V_{m}, m \geq 1
\end{aligned}
$$

Por argumentos de densidad

$$
\begin{aligned}
& \left.\int_{0}^{T}\left[b_{\alpha}\left(u^{\prime \prime}(t), v\right)\right)+\psi(t) b_{\alpha}(S u(t), v(t))\right] d t=\int_{0}^{T} b_{\alpha}(h(t), v(t)) d t \\
& \forall v \in L^{2}\left(0, T ; D\left(S^{\alpha}\right)\right)
\end{aligned}
$$

lo que demuestra la primera parte del Teorema 1. 
Para demostrar que la solución $u$ verifica el estimado de energía (3.14), tendremos en cuenta que por el Lema 1, se tiene las siguientes convergencias:

$$
\begin{aligned}
& u_{m} \rightarrow u \text { fuerte } C^{0}\left([0, T] ; D\left(S^{(2 \alpha+1) / 2}\right)\right) \\
& u_{m}^{\prime} \rightarrow u^{\prime} \text { fuerte } C^{0}\left([0, T] ; D\left(S^{(4 \alpha+1) / 4}\right)\right)
\end{aligned}
$$

A continuación demostramos que:

$$
\begin{array}{ll}
u_{m}(t) \rightarrow u(t) \text { débil en } D\left(S^{\alpha+1}\right) & \forall t \in[0, T] \\
u_{m}^{\prime}(t) \rightarrow u^{\prime}(t) \text { débil en } D\left(S^{(2 \alpha+1) / 2}\right) & \forall t \in[0, T]
\end{array}
$$

En efecto, sea $v \in V_{k} ; k \geq m$. Tenemos

$$
\begin{aligned}
\left(S^{\alpha+1} u_{m}(t), S^{\alpha+1} v\right)=\left(S^{(2 \alpha+1) / 2} u_{m}(t), S^{(4 \alpha+3) / 4} v\right) & \rightarrow\left(S^{(2 \alpha+1) / 2} u(t), S^{(4 \alpha+3) / 4} v\right)= \\
& =\left(S^{\alpha+1} u(t), S^{\alpha+1} v\right)
\end{aligned}
$$

desde que por (3.41)

$\mid\left(S^{(2 \alpha+1) / 2} u_{m}(t)-S^{(2 \alpha+1) / 2} u(t), S^{(4 \alpha+3) / 4} v|\leq| S^{(2 \alpha+1) / 2} u_{m}(t)-S^{(2 \alpha+1) / 2} u(t) \| S^{(4 \alpha+3) / 4} v \mid \stackrel{m \rightarrow \infty}{\longrightarrow} 0\right.$

nuevamente por argumentos de densidad, la convergencia en (3.44) se verfica para todo $v \in D\left(S^{\alpha+1}\right)$. Esto demuestra (3.41).

Análogamente:

$$
\begin{aligned}
\left(S^{(2 \alpha+1) / 2} u_{m}^{\prime}(t), A^{(2 \alpha+1) / 2} v\right)=\left(S^{(4 \alpha+1) / 4} u_{m}^{\prime}(t), S^{(4 \alpha+3) / 4} v\right) & \rightarrow\left(S^{(4 \alpha+1) / 4} u^{\prime}(t), S^{(4 \alpha+3) / 4} v\right)= \\
& =\left(S^{(2 \alpha+1) / 2} u^{\prime}(t), S^{(2 \alpha+1) / 2} v\right)
\end{aligned}
$$

Luego por el Estimado 1 y (3.41), (3.42), obtenemos

$$
\left|S^{(2 \alpha+1) / 2} u^{\prime}(t)\right|^{2}+\left|S^{\alpha+1} u(t)\right|^{2} \leq \varliminf_{m \rightarrow \infty}\left\{\left|S^{(2 \alpha+1) / 2} u_{m}^{\prime}(t)\right|^{2}+\left|S^{\alpha+1} u_{m}(t)\right|^{2}\right\} \leq D_{0} e^{D_{1} t}
$$

Unicidad. Sean $u, z$ dos soluciones de (3.3) y $y=u-z$. Entonces

$$
\begin{aligned}
& y \in L^{\infty}\left(0, T ; D\left(S^{\alpha+1}\right)\right) \\
& y^{\prime} \in L^{\infty}\left(0, T ; D\left(S^{(2 \alpha+1) / 2}\right)\right) \\
& y^{\prime \prime} \in L^{2}\left(0, T ; D\left(S^{\alpha}\right)\right) \\
& y(0)=0 ; \quad y^{\prime}(0)=0
\end{aligned}
$$

y satisface la ecuación

$$
B y^{\prime \prime}+\psi(t) A y=0 \quad \text { en } L^{2}\left(0, T ; D\left(S^{\alpha}\right)\right)
$$

Componiendo con $w=2 y^{\prime}(t) \in D\left(S^{\alpha}\right)$ en esta ecuación, obtenemos 


$$
\frac{d}{d t}\left\{\left|S^{\alpha} y^{\prime}(t)\right|^{2}+\psi(t)\left|S^{(2 \alpha+1) / 2} y(t)\right|^{2}\right\}=\psi^{\prime}(t)\left|S^{(2 \alpha+1) / 2} y(t)\right|^{2} \leq M_{1}\left|S^{(2 \alpha+1) / 2} y(t)\right|^{2}
$$

integrando de 0 a t y teniendo en cuenta que $y(0)=y^{\prime}(0)=0$

$$
\eta(t)=\left|S^{\alpha} y^{\prime}(t)\right|^{2}+m_{0}\left|S^{(2 \alpha+1) / 2} y(t)\right|^{2} \leq C \int_{0}^{t} \eta(s) d s
$$

y por el lema de Gronwall $S^{\alpha} y^{\prime}(t)=S^{(2 \alpha+1) / 2} y(t)=0 \quad \forall t \in[0, T]$; luego $u=z$.

4. Problema No-Lineal (Solución local). En esta parte demostraremos la existencia de solución local del problema no-lineal:

$$
\begin{aligned}
& B u^{\prime \prime}+M\left(|u(t)|_{W}\right) A u=f \\
& u(0)=u_{0} ; u^{\prime}(0)=u_{1}
\end{aligned}
$$

donde:

$W$ es un espacio de Banach, real, reflexivo, tal que su correspondiente espacio dual $W^{*}$ es estrictamente convexo.

$$
D\left(S^{(2 \alpha+1) / 2}\right) \subset W
$$

Entonces de (4.4) y de la teoría de los operadores máximo monótonos, obtenemos las siguientes propiedades del operador de dualidad sobre $W$.

La aplicación de dualidad $J: W \rightarrow W^{*}$ es simple valuada, demicontinua, máximo monótona, acotada, coercitiva y

$$
|J u|_{W^{*}}=|u|_{W}
$$

La norma $u \rightarrow|u|_{W}$ es G-diferenciable sobre $W-\{0\}$ y si $\psi(u)=|u|_{W}$, entonces

$$
\psi^{\prime}(u)=\frac{J u}{|u|_{W}} \forall u \neq 0
$$

\section{Hipótesis sobre la Función $M$}

H-1 $\quad M \in C^{1}\left([0, T] ; \mathbb{R}^{+}\right)$, donde $T$ es un número real positivo.

H-2 $M(0)=0 ; \exists \varepsilon>0$, tal que $M(s)>0 \quad \forall s \in\langle 0, \varepsilon\rangle$.

H-3 $\quad M^{\prime}(s) \geq 0 \quad \forall s \in[0, T]$. 
Definición de Constantes. A continuación definimos diversas constantes que aparecen en esta parte del trabajo.

Para datos iniciales $0 \neq u_{0}, u_{1}, f$ convenientes, consideramos:

C-1

$$
0<m_{0}=M\left(\frac{\left|u_{0}\right|_{W}}{2}\right)
$$

C-2 $\quad C_{0}=\left|S^{(2 \alpha+1) / 2} u_{1}\right|^{2}+M\left(\left|u_{0}\right|_{W}\right)\left|S^{\alpha+1} u_{0}\right|^{2}+|f|_{L^{2}\left(0, T ; D\left(A^{(2 \alpha+1) / 2}\right)\right)}^{2}$

C-3 $\quad C_{1}=\min \left\{1, m_{0}\right\}$

C-4 $\quad D_{0}=\frac{C_{0}}{C_{1}}$

C-5 $0<K^{2}=2 D_{0} \leq 1$

C-6 $\quad|v|_{W} \leq d_{2}\left|S^{(2 \alpha+1) / 2} v\right| \quad \forall v \in D\left(S^{(2 \alpha+1) / 2}\right)$

C-7 $\quad M_{0}=\operatorname{Sup}\left\{M^{\prime}(s) / s \in\left[0, d_{2} K\right]\right\}$

C-8 $\quad C_{2}=\operatorname{Max}\left\{1, M_{0}\right\}$

C-9 $\quad D_{1}=\frac{C_{2}}{C_{1}}$

C-10 $\quad|v|_{W} \leq d_{2}\left|S^{(2 \alpha+1) / 2} v\right| \quad \forall v \in D\left(S^{(2 \alpha+1) / 2}\right)$

C-11 $\quad 0<T^{*}=\frac{\left|u_{0}\right|_{W}}{2 d_{2} K}$

C-12 $T_{0}=\min \left\{T, T^{*}, \frac{1}{D_{1}}\right\}$

Consideremos el siguiente conjunto:

$$
G=\left\{\begin{array}{lr}
v \in L^{\infty}\left(0, T_{0} ; D\left(S^{\alpha+1}\right)\right) / v^{\prime} \in L^{\infty}\left(0, T_{0} ; D\left(S^{2 \alpha+1 / 2}\right)\right), & v^{\prime \prime} \in L^{2}\left(0, T_{0} ; D\left(S^{\alpha}\right)\right) \\
v(0)=u_{0} \neq 0 ; & \left|S^{(2 \alpha+1) / 2} v^{\prime}(t)\right|^{2}+\left|S^{\alpha+1} v(t)\right|^{2} \leq K^{2} \quad \forall t \in\left[0, T_{0}\right]
\end{array}\right\}
$$

Lema 2. Si $v \in G$ y $\psi(t)=M\left(|v(t)|_{W}\right)$, entonces

$$
\begin{aligned}
& 0<m_{0} \leq \psi(t) \quad \forall t \in\left[0, T^{*}\right] \\
& \left|\psi^{\prime}(t)\right| \leq M_{0} d_{2}=M_{1} \quad \forall t \in\left[0, T^{*}\right]
\end{aligned}
$$

Demostración. Sea $v \in G$, entonces

$$
\left.|| v(t)\right|_{W}-|v(0)|_{W}|\leq| v(t)-\left.v(0)\right|_{W}=\left|\int_{0}^{t} v^{\prime}(s) d s\right|_{W} \leq \int_{0}^{t}\left|v^{\prime}(s)\right|_{W} d s \leq d_{2} K t
$$


De esta desigualdad obtenemos

$$
\left|u_{0}\right|_{W}-d_{2} K t=|v(0)|_{W}-d_{2} K t \leq|v(t)|_{W}
$$

Luego

$$
0<\frac{\left|u_{0}\right|_{W}}{2}=\left|u_{0}\right|_{W}-\frac{\left|u_{0}\right|_{W}}{2} \leq\left|u_{0}\right|_{W}-d_{2} K T^{*} \leq\left|u_{0}\right|_{W}-d_{2} K t \leq|v(t)|_{W} \quad \forall t \in\left[0, T^{*}\right]
$$

Por la hipótesis H-2

$$
\psi(t)=M\left(|v(t)|_{W}\right) \geq M\left(\frac{\left|u_{0}\right|_{W}}{2}\right)=m_{0}>0
$$

lo que demuestra (4.5).

Debemos ahora obtener estimados para $\psi^{\prime}(t)$. Tenemos por la regla de la cadena, que

$$
\psi^{\prime}(t)=M^{\prime}\left(|v(t)|_{W}\right)\left\langle\frac{J v(t)}{|v(t)|_{W}}, v^{\prime}(t)\right\rangle_{W^{*} \times W}
$$

Entonces

$$
\left|\psi^{\prime}(t)\right| \leq\left|M^{\prime}\left(|v(t)|_{W}\right)\right| \frac{|J v(t)|_{W^{*}}}{|v(t)|_{W}}\left|v^{\prime}(t)\right|_{W} \leq M_{0}\left|v^{\prime}(t)\right|_{W} \leq M_{0} d_{2}\left|A^{(2 \alpha+1) / 2} v^{\prime}(t)\right| \leq M_{0} d_{2}=M_{1}
$$

lo que demuestra el Lema 2.

Teorema 2. Sean

$$
\begin{aligned}
& v \in G \\
& 0 \neq u_{0} \in D\left(S^{\alpha+1}\right) \\
& u_{\}} \in D\left(S^{(2 \alpha+1) / 2}\right) \\
& f \in L^{2}\left(0, T_{0} ; D\left(S^{(2 \alpha+1) / 2}\right)\right)
\end{aligned}
$$

Entonces, existe una única solución $u \in G$ del problema:

$$
\begin{aligned}
& B u^{\prime \prime}+M\left(|v(t)|_{W}\right) A u=f \quad \text { en } L^{2}\left(0, T_{0} ; D\left(S^{\alpha}\right)\right) \\
& u(0)=u_{0} ; u^{\prime}(0)=u_{\mathrm{I}}
\end{aligned}
$$

Demostración: Procediendo como en la demostración del Teorema 1, haciendo $T=T_{0}, \psi(t)=M\left(|v(t)|_{W}\right)$ y utilizando el Lema 1 , se obtiene que para todo $v \in G$, existe una única función $u$, tal que: 


$$
\begin{aligned}
& u \in L^{\infty}\left(0, T_{0} ; D\left(S^{\alpha+1}\right)\right) \\
& u^{\prime} \in L^{\infty}\left(0, T_{0} ; D\left(S^{(2 \alpha+1) / 2}\right)\right) \\
& u^{\prime \prime} \in L^{2}\left(0, T_{0} ; D\left(S^{\alpha}\right)\right) \\
& B u^{\prime \prime}+\psi(t) A u=f \quad \text { en } L^{2}\left(0, T ; D\left(S^{\alpha}\right)\right)
\end{aligned}
$$

y además satisface el siguiente estimado de energía:

$$
\left|S^{(2 \alpha+1) / 2} u^{\prime}(t)\right|^{2}+\left|S^{\alpha+1} u(t)\right|^{2} \leq D_{0} \exp \left(D_{1} t\right) \leq K^{2}
$$

Luego $u \in G$.

Teorema 3. Sean $u_{0}, u_{1}, f$ datos iniciales que satisfacen las condiciones del Teorema 2. Entonces existe una única solución $u \in G$ del problema (4.11) y (4.12).

Demostración. La idea es resolver una sucesión de problemas de la forma

$$
\begin{aligned}
& B z_{p}^{\prime \prime}+M\left(\left|z_{p-1}(t)\right|_{W}\right) A z_{p}=f \text { en } L^{2}\left(0, T_{0} ; D\left(S^{\alpha}\right)\right) \\
& z_{p}(0)=u_{0} ; z_{p}^{\prime}(0)=u_{1} \\
& p \geq 2
\end{aligned}
$$

donde:

$z_{2}$ es la única solución del problema

$$
\begin{aligned}
& B z_{2}^{\prime \prime}+M\left(\left|u_{0}\right|_{W}\right) A z_{2}=f \text { en } L^{2}\left(0, T_{0} ; D\left(S^{\alpha}\right)\right) \\
& z_{2}(0)=u_{0} ; z_{2}^{\prime}(0)=u_{1}
\end{aligned}
$$

Desde el Teorema 2, podemos definir una función $S: G \rightarrow G$ tal que $S z_{p-1}=z_{p}, p \geq 3$ donde $z_{p}$ es la única solución del problema (4.18).

La dificultad principal es demostrar la convergencia

$$
M\left(\left|z_{p-1}(t)\right|_{W}\right) A z_{p} \stackrel{m \rightarrow \infty}{\longrightarrow} M\left(|z(t)|_{W}\right) A z \text { débil en } L^{2}\left(0, T_{0} ; D\left(S^{\alpha}\right)\right)
$$

En primer lugar probaremos que

$$
M\left(\left|z_{p-1}\right|_{W}\right) \stackrel{m \rightarrow \infty}{\longrightarrow} M\left(|z|_{W}\right) \text { en } C^{0}\left(\left[0, T_{0}\right]\right)
$$

En efecto;

desde que la sucesión $\left(z_{p}\right)_{p \geqslant 2} \subset G$, obtenemos que existe una función $z \in L^{\infty}\left(0, T_{0} ; D\left(S^{\alpha+1}\right)\right)$ tal que:

$$
\begin{aligned}
& z_{p} \rightarrow z \text { débil * en } L^{\infty}\left(0, T_{0} ; D\left(S^{\alpha+1}\right)\right) \\
& z_{p}^{\prime \prime} \rightarrow z^{\prime \prime} \text { débil en } L^{2}\left(0, T_{0}^{\prime} ; D\left(S^{\alpha}\right)\right)
\end{aligned}
$$


De (4.25) y como $B$ es compacto

$$
B z_{p}^{\prime \prime} \rightarrow B z^{\prime \prime} \text { débil en } L^{2}\left(0, T_{0} ; D\left(S^{\alpha}\right)\right)
$$

además por el Lema 2

$$
z_{p-1} \stackrel{p \rightarrow \infty}{\longrightarrow} z \text { en } C\left(\left[0, T_{0}\right] ; D\left(A^{\eta+(2 \alpha+1) / 2}\right)\right) \subset C\left(\left[0, T_{0}\right] ; W\right) ; \eta \in[0,1 / 2)
$$

Ahora por la hipótesis $\mathrm{H}-1$, sobre la función $M$

$$
\begin{aligned}
& M\left(s_{1}\right)-M\left(s_{2}\right)=M^{\prime}(\theta)\left(s_{1}-s_{2}\right) \\
& \forall s_{1}, s_{2}, \quad \theta \in\left[s_{1}, s_{2}\right]
\end{aligned}
$$

Entonces

$$
\begin{aligned}
& \left|M\left(\left|z_{p-1}(t)\right|_{W}\right)-M\left(|z(t)|_{W}\right)\right| \leq \\
& \leq\left|M^{\prime}(\theta(t))\left(\left|z_{p-1}(t)\right|_{W}-|z(t)|_{W}\right)\right| \leq C\left|z_{p-1}(t)-z(t)\right|_{W} \stackrel{p \rightarrow \infty}{\longrightarrow} 0
\end{aligned}
$$

donde $\theta(t)=\beta\left|z_{p-1}(t)\right|_{W}+(1-\beta)|z(t)|_{W} \leq d_{2} K$ por

Por otro lado:

$$
\begin{aligned}
& \int_{0}^{T}\left|\left(M\left(\left|z_{p-1}(t)\right|_{W}\right) A z_{p}(t)-M\left(|z(t)|_{W}\right) A z(t), v(t)\right)_{\alpha}\right| d t \\
= & \int_{0}^{T}\left|\left(M\left(\left|z_{p-1}(t)\right|_{W}\right) A^{\alpha+1} z_{p}(t)-M\left(|z(t)|_{W}\right) A^{\alpha+1} z(t), A^{\alpha} v(t)\right)\right| d t \\
\leq & \int_{0}^{T}\left|\left(M\left(\left|z_{p-1}(t)\right|_{W}\right) A^{\alpha+1} z_{p}(t)-M\left(\left|z_{p-1}(t)\right|_{W}\right) A^{\alpha+1} z(t), A^{\alpha} v(t)\right)\right| d t+ \\
+ & \int_{0}^{T}\left|\left(M\left(\left|z_{p-1}(t)\right|_{W}\right) A^{\alpha+1} z(t)-M\left(|z(t)|_{W}\right) A^{\alpha+1} z(t), A^{\alpha} v(t)\right)\right| d t \leq \\
\leq & \int_{0}^{T}\left|M\left(\left|z_{p-1}(t)\right|_{W}\right)\right|\left|\left(A^{\alpha+1} z_{p}(t)-A^{\alpha+1} z(t), A^{\alpha} v(t)\right)\right| d t+ \\
& +\int_{0}^{T}\left|M\left(\left|z_{p-1}(t)\right|_{W}\right)-M\left(|z(t)|_{W}\right)\right|\left|A^{\alpha+1} z(t)\right| A^{\alpha} v(t) \mid d t \leq \\
& \leq C \int_{0}^{T}\left|\left(A^{\alpha+1} z_{p}(t)-A^{\alpha+1} z(t), A^{\alpha} v(t)\right)\right| d t+ \\
& +C \int_{0}^{T}\left|M\left(\left|z_{p-1}(t)\right|_{W}\right)-M\left(|z(t)|_{W}\right)\right| d t \stackrel{p \rightarrow \infty}{\longrightarrow} 0
\end{aligned}
$$




$$
B z^{n}+M\left(|z(t)|_{W}\right) A z=f \quad \text { en } \quad L^{2}\left(0, T ; D\left(S^{\alpha}\right)\right)
$$

Procediendo como en la demostración del Teorema 1, se prueba que:

$$
\begin{aligned}
& z_{p}(t) \rightarrow z(t) \text { débil en } D\left(S^{\alpha+1}\right) \quad \forall t \in\left[0, T_{0}\right] \\
& z_{p}^{\prime}(t) \rightarrow z^{\prime}(t) \text { débil en } D\left(S^{(2 \alpha+1) / 2}\right) \quad \forall t \in\left[0, T_{0}\right]
\end{aligned}
$$

Entonces

$$
\left|S^{(2 \alpha+1) / 2} z^{\prime}(t)\right|^{2}+\left|S^{\alpha+1} z(t)\right|^{2} \leq \frac{\lim }{p \rightarrow \infty}\left\{\left|S^{(2 \alpha+1) / 2} z_{p}^{\prime}(t)\right|^{2}+\left|S^{\alpha+1} z_{p}(t)\right|^{2}\right\} \leq K^{2}
$$

Por lo tanto $z \in G$.

\section{Unicidad.}

Sean $u, z$ dos soluciones del problema (4.11) y (4.12) y $y=u-z$. Entonces se verifica que:

$$
\begin{aligned}
& y \in L^{\infty}\left(0, T_{0} ; D\left(S^{\alpha+1}\right)\right) \\
& y^{\prime} \in L^{\infty}\left(0, T_{0} ; D\left(S^{2 \alpha+1 / 2}\right)\right) \\
& y^{\prime \prime} \in L^{\infty}\left(0, T_{0} ; D\left(S^{\alpha}\right)\right) \\
& y(0)=y^{\prime}(0)=0
\end{aligned}
$$

y satisface la ecuación

$$
B y^{\prime \prime}+M\left(|u(t)|_{W}\right) A y=\left(M\left(|z(t)|_{W}\right)-M\left(|u(t)|_{W}\right)\right) A z \text { en } L^{2}\left(0, T_{0} ; D\left(S^{\alpha}\right)\right)
$$

Componiendo con $w(t)=2 y^{\prime}(t)$ en esta ecuación y teniendo en cuenta que

$$
\left|M\left(|z(t)|_{W}\right)-M\left(|u(t)|_{W}\right)\right| \leq C|z(t)-u(t)|_{W}=C|y(t)|_{W}
$$

obtenemos

$$
\begin{aligned}
\frac{d}{d t}\left\{\left|S^{\alpha} y^{\prime}(t)\right|^{2}+\psi(t)\right. & \left.\left|S^{(2 \alpha+1) / 2} y(t)\right|^{2}\right\} \leq \\
& \leq C|y(t)|_{0}\left|S^{\alpha+1} z(t)\right|\left|S^{\alpha} y^{\prime}(t)\right|+\psi^{\prime}(t)\left|S^{(2 \alpha+1) / 2} y(t)\right|^{2} \leq \\
& \leq C\left|S^{(2 \alpha+1) / 2} y(t)\right|\left|S^{\alpha} y^{\prime}(t)\right|+C\left|S^{(2 \alpha+1) / 2} y(t)\right|^{2} \leq \\
& \leq C\left|S^{\alpha} y^{\prime}(t)\right|+C\left|S^{(2 \alpha+1) / 2} y(t)\right|^{2}
\end{aligned}
$$

donde $C$ representa diversas constantes que no dependen de $t$. Integrando de 0 a $t$ y teniendo en cuenta que $y(0)=y^{\prime}(0)=0$ obtenemos 


$$
\eta(t)=\left|S^{\alpha} y^{\prime}(t)\right|^{2}+m_{0}\left|S^{(2 \alpha+1) / 2} y(t)\right|^{2} \leq C \int_{0}^{t} \eta(s) d s
$$

y por el lema de Gronwall $S^{\alpha} y^{\prime}(t)=S^{(2 \alpha+1) / 2} y(t)=0 \quad \forall t \in\left[0, T_{0}\right]$; luego $u=z$.

\section{Aplicaciones}

1. Consideremos las siguientes hipótesis.

(H 1) $\left(V,(u, v)_{V}\right)$, es un espacio de Hilbert real, separable.

(H 2) Las formas bilineales $a, b: V \times V \rightarrow \mathbb{R}$ son acotadas y simétricas. Asimismo $a(u, v)$ es fuertemente monótona, y $b(u, v)$ es compacta y estrictamente positiva.

Se tiene el siguiente teorema.

Teorema. Supongamos se cumplen las hipótesis H 1, H 2. Entonces

existe un sistema ortogonal completo $\left\{w_{j}\right\}_{j \geq 1}$ de autovalores en $V$ con respecto al producto escalar $(u, v)=a(u, v), \mathrm{y}$ una familia de autovectores $\left\{\lambda_{j}\right\}_{j \geq 1}$ de multiplicidad finita para cada $\lambda_{k}$, tales que:

$$
\begin{aligned}
& a\left(w_{j}, v\right)=\lambda_{j} b\left(w_{j}, v\right) \quad \forall v \in V \\
& b\left(w_{j}, w_{i}\right)=\delta_{j i} \quad \forall i, j \\
& u=\sum_{j=1}^{\infty} b\left(u, w_{j}\right) w_{j} \quad \forall u \in V \\
& 0<\lambda_{1} \leq \lambda_{2} \leq \ldots \ldots \ldots . . ., \quad \lambda_{k} \stackrel{k \rightarrow \infty}{\longrightarrow} \infty
\end{aligned}
$$

1. Sea $\Omega \subset \mathbb{R}^{n}$ un abierto regular. Consideremos:

$V=H_{0}^{1}(\Omega), W=L^{p}(\Omega), H=L^{2}(\Omega)$

$1<p \leq \frac{2 n}{n-2}$, si $n \geq 3, y 1<p<\infty$, si $n=1,2$

$M(s)=a+b s^{q}, q \geq 1, a \geq 0, b>0$

$\rho \in L^{\infty}(\Omega) ; 0<\rho(x) \leq \rho_{1}$, c.t.p. de $\Omega$.

En este caso, un cálculo directo demuestra que el operador de dualidad $J: W \rightarrow W^{\prime}$ esta definido por

$$
J(u)=|u|_{p}^{2-p}|u|^{p-2} u ; 1<p<\infty
$$


Entonces existe una única solución local del problema

$$
\begin{cases}\rho(x) \frac{\partial^{2} u}{\partial t^{2}}+\left(a+b\left(\int_{\Omega}|u(x, t)|^{p}\right)^{q / p}\right)(-\Delta u)=f & \text { en } Q \\ u=0 & \text { en } \Gamma \\ u(0)=u_{0} ; u^{\prime}(0)=u_{1} & \text { en } \Omega\end{cases}
$$

2. Sea $M(s)=N\left(s^{2}\right)$ y $J: W \rightarrow W^{*}$ el operador de dualidad sobre $W$. Consideremos el problema:

$$
\left\{\begin{array}{l}
B u^{\prime \prime}+N\left(|u|_{W}^{2}\right) A u=f \\
u(0)=u_{0} ; u^{\prime}(0)=u_{1}
\end{array}\right.
$$

en este caso si $\varphi(u)=|u|_{W}^{2}$, entonces $\varphi^{\prime}(u)=2 J(u), \forall u \in W$, lo que facilita los cálculos desde que no requiere que $u \neq 0$.

En el caso particular que $W$ sea un espacio de Hilbert $\varphi^{\prime}(u)=2 J u$, donde $J$ es el operador de Riesz, $\langle J u, v\rangle_{W * \times W}=(u, v)_{W} ; \forall u, v \in W$.

Como casos particulares de la aplicación 2, para $W=V=D\left(A^{1 / 2}\right)$, tenemos el problema abstracto de Kirchhoff:

$$
\left\{\begin{array}{l}
B u^{\prime \prime}+N\left(|u|_{V}^{2}\right) A u=f \\
u(0)=u_{0} ; u^{\prime}(0)=u_{1}
\end{array}\right.
$$

formulado por Lions, J.L. en [9]. Si $W=H$, se tiene el problema de Carrier abstracto:

$$
\left\{\begin{array}{l}
B u^{\prime \prime}+N\left(|u|_{H}^{2}\right) A u=f \\
u(0)=u_{0} ; u^{\prime}(0)=u_{1}
\end{array}\right.
$$

Una adecuada generalización de las aplicaciones (5.6), (5.7) es considerar $W=D\left(A^{\alpha}\right)$ para obtener:

$$
\left\{\begin{array}{l}
B u^{\prime \prime}+N\left(|u|_{\alpha}^{2}\right) A u=f \\
u(0)=u_{0} ; u^{\prime}(0)=u_{l}
\end{array}\right.
$$

conocido como el modelo abstracto de Kirchhoff - Carrier. 


\section{REFERENCIAS BIBLIOGRÁFICAS}

[1] AROSIO A -SPAGNOLO S.- Global solutions of the Cauchy problem for a non-linear Hyperbolic Equation. Universita di Pisa. Departamento de Matemática. Roma (1982).

[2] CARRIER G.F. On the non-linear vibration problem of the elastic string. Quart. Appl. Math. - 3- (1945).

[3] COUSIN A., FROTA C., LARKIN N., MEDEIROS L. A. On the abstrac model of KirchhoffCarrier Equation. Comm. In App. Analysis. - 3 - (1997).

[4] CRIPPA H. On Local Solutions of Some mildly Degenerate Hyperbolic Equations. Nonlinear Analysis, Vol. 21 (8) (1993).

[5] EBIHARA Y. MEDEIROS L.A. -MILLA. M- Local Solutions for a nonlinear degenerate hyperbolic equations. Nonlinear Analysis. Vol. 10 (1986).

[6] IZAGUIRRE R., VÉLIZ V. Solución local para una clase de ecuaciones no-lineales degeneradas tipo Kirchhoff - Carrier. I Seminario Internacional de Ecuaciones Diferenciales y Aplicaciones. Universidad Ricardo Palma - Lima - Perú - (1999).

[7] IZAGUIRRE R., VÉLIZ V. Solución Local para una clase de ecuaciones no-lineales de tipo Kirchhoff. Actas del $45^{\circ}$ Seminario Brasileiro de Analise (1997).

[8] KIRCHHOFF G. Vorlesungenuber mechanik. Teubner, Leipzig (1883).

[9] LIONS J.L. Quelques Methodes de Resolution des Probleme aux limites nonlinear. Dunod. Paris. (1969).

[10] MEDEROS L.A., MLLA M. Solutions for the Equation of Nonlinear Vibrations Sobolev Spaces of Fractionary Order. Math. Apl. Comp. (1987).

[11] MEDEIROS L.A., MLLA M. Remarks on a nonlinear model vibrations of string with damping. 30 Seminario Brasilero de Analise L N C C R J. (1989).

[12] ONO K. Global existence, Decay and Blowup of Solutions for Some Mildly Degenerate Nonlinear Kirchhoff String. J. Diff. Eq. 137 (1997).

[13] PERLA G. On classical solutions of a quasilinear hyperbolic equations. Nonlinear Analysis. Vol. 3(1979).

[14] POHOZAEV S. The Kirchhoff quasilinear hyperbolic equation. Differenctial Equations Vol. 21. (1985).

[15] POHOZAEV S. On a class of quasilinear hyperbolic equation. Math. Sbornik, Vol. 96 (1975).

[16] RIVERA P. On local strong solutions of a nonlinear partial differential equation. Appl. Analysis Vol. 10. (1980).

[17] SIMON J. Compact Sets in the Space $L^{p}(0, T ; B)$. Universite Pierre et Marie Curie. Laboratoire d'Analyse Numerique (1985).

[18] YAMADA Y. Some Nonlinear Degenerate Wave Equations. Non Linear Analysis, 10 (11) (1987).

[19] ZEIDLER E. Non Linear Functional Analysis. Part II-B. (1990). 